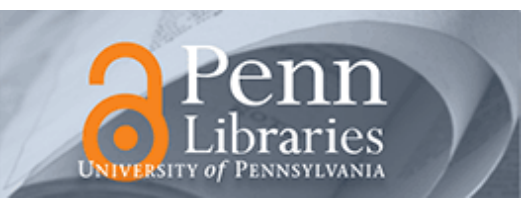

University of Pennsylvania

ScholarlyCommons

July 2008

\title{
Three-Dimensional Plasmonic Nanoswitch: Extreme Variation of Scattering Properties upon Rotation
}

\author{
Andrea Alù \\ University of Pennsylvania, andreaal@seas.upenn.edu \\ Nader Engheta \\ University of Pennsylvania, engheta@seas.upenn.edu
}

Follow this and additional works at: https://repository.upenn.edu/ese_papers

\section{Recommended Citation \\ Andrea Alù and Nader Engheta, "Three-Dimensional Plasmonic Nanoswitch: Extreme Variation of Scattering Properties upon Rotation", . July 2008.}

Copyright 2008 IEEE. Reprinted from Proceedings of the Antennae and Propagation Society International Symposium, APS 2008, 4 pages.

This material is posted here with permission of the IEEE. Such permission of the IEEE does not in any way imply IEEE endorsement of any of the University of Pennsylvania's products or services. Internal or personal use of this material is permitted. However, permission to reprint/republish this material for advertising or promotional purposes or for creating new collective works for resale or redistribution must be obtained from the IEEE by writing to pubs-permissions@ieee.org. By choosing to view this document, you agree to all provisions of the copyright laws protecting it.

This paper is posted at ScholarlyCommons. https://repository.upenn.edu/ese_papers/459

For more information, please contact repository@pobox.upenn.edu. 


\title{
Three-Dimensional Plasmonic Nanoswitch: Extreme Variation of Scattering Properties upon Rotation
}

\begin{abstract}
The anomalous interaction of light with plasmonic materials has fascinated scientists and non-scientists for centuries. The recent interest in research on anomalous resonant phenomena involving plasmonic nanoparticles may be associated with the relevant advancements in nanotechnology of the last decade, which allow realization of artificial materials with a tailored anomalous electromagnetic response, with large design flexibility. We have recently proposed several novel applications that involve plasmonic nanoparticles, employed as nanoantennas, nanocircuit elements or nanowaveguides. In particular, in the framework of our recent paradigm for scaling the circuit concepts to optical frequencies [1], we have suggested that the combination of plasmonic and non-plasmonic nanoparticles may give rise to a novel type of circuitry at frequencies (IR and optical) at which metals lose their highly conductive properties. In this context, we have proposed the synthesis and design of nanotransmission-lines [2-3] and backwardwave nanomaterials [4], the optical equivalent of connecting "shorting" wires [5], combinations of parallel and series nanocircuit elements [6-7] and basic collections of nanofilters [8].

\section{Comments}

Copyright 2008 IEEE. Reprinted from Proceedings of the Antennae and Propagation Society International Symposium, APS 2008, 4 pages.

This material is posted here with permission of the IEEE. Such permission of the IEEE does not in any way imply IEEE endorsement of any of the University of Pennsylvania's products or services. Internal or personal use of this material is permitted. However, permission to reprint/republish this material for advertising or promotional purposes or for creating new collective works for resale or redistribution must be obtained from the IEEE by writing to pubs-permissions@ieee.org. By choosing to view this document, you agree to all provisions of the copyright laws protecting it.
\end{abstract}




\title{
Three-Dimensional Plasmonic Nanoswitch: Extreme Variation of Scattering Properties upon Rotation
}

\author{
Andrea Alù and Nader Engheta \\ Dept. of Electrical and Systems Engineering, University of Pennsylvania \\ 200 South $33^{\text {rd }}$ St., Philadelphia, PA 19104, U.S.A. \\ andreaal, engheta@ee.upenn.edu
}

\section{Introduction}

The anomalous interaction of light with plasmonic materials has fascinated scientists and non-scientists for centuries. The recent interest in research on anomalous resonant phenomena involving plasmonic nanoparticles may be associated with the relevant advancements in nanotechnology of the last decade, which allow realization of artificial materials with a tailored anomalous electromagnetic response, with large design flexibility. We have recently proposed several novel applications that involve plasmonic nanoparticles, employed as nanoantennas, nanocircuit elements or nanowaveguides. In particular, in the framework of our recent paradigm for scaling the circuit concepts to optical frequencies [1], we have suggested that the combination of plasmonic and non-plasmonic nanoparticles may give rise to a novel type of circuitry at frequencies (IR and optical) at which metals lose their highly conductive properties. In this context, we have proposed the synthesis and design of nanotransmission-lines [2-3] and backward-wave nanomaterials [4], the optical equivalent of connecting "shorting" wires [5], combinations of parallel and series nanocircuit elements [6-7] and basic collections of nanofilters [8].

Applying these concepts, one may envision and tailor combinations of nanoparticles that provide a complex response at will, by simply applying basic circuit rules [1-9]. As a special example of these possibilities, here we describe the anomalous scattering properties of a three-dimensional (3-D) resonant nanoparticle obtained by conjoining together two hemispheres with "complementary" electromagnetic properties. In this way, analogous to the twodimensional case of conjoined half-cylinders we have studied earlier [6-7], we realize a simple resonant interconnection between two basic nanocircuit elements in 3-D, showing how, depending on the orientation of the impinging electric field, its response may switch from a "short" to an "open" circuit through all the possible intermediate stages. From a scattering point of view, this composite particle may represent a basic 3-D nanoswitch that may effectively pass from resembling a perfect electric to perfect magnetic nanosphere after a simple rotation of 90 degrees. We provide here some physical insights into the anomalous optical response of this 3-D nanostructure and its interpretation in terms of nanocircuit theory. For the interested reader, we have discussed in details the theoretical findings associated with this electromagnetic problem in [10]. 


\section{Extreme Scattering Variation Upon Rotation for 3-D nanospheres}

Consider the geometry depicted in Fig. 1, consisting of a three-dimensional (3-D) nanosphere composed of two conjoined hemispheres of permittivity $\varepsilon_{1}$ and $\varepsilon_{2}$, respectively. We assume that the nanoparticle has a radius much smaller than the wavelength of operation, so that a "small-radius, quasi-static" analysis may apply. Following our findings in [9], despite the complexity of the problem it may be shown that, in the special case for which $\varepsilon_{1}=-\varepsilon_{2}$, independent of the background permittivity, the problem may be solved analytically in closed-form for the potential and field distribution inside and outside this 3-D spherical nanoparticle, for any orientation of the impinging electric field. This is analogous to what we obtained for the 2-D case of cylinders [6]. Figure $1 \mathrm{a}$ and $1 \mathrm{~b}$ report the potential distributions for electric field parallel and normal to the common interface (the black arrows in the figure indicate the polarization of the impressed electric field).

When the field is parallel to the interface (panel a) the hemispheres are in a parallel interconnection, following our findings in [6-7] for the two-dimensional case (2-D). Likewise, in the configuration of panel $b$ they are connected in series. Since they are at resonance for $\varepsilon_{1}=-\varepsilon_{2}$, consistent with a resonant pair of circuit elements, they behave respectively as a perfectly magnetic and a perfectly electric homogeneous nanosphere from a scattering point of view, consistent with an open- and a short-circuit configuration, respectively. It can indeed be seen in Fig. 1 how a simple rotation of the external electric field (black arrows in the figure) produces an extreme variation of the scattering response of the nanoparticle, with the external potential distribution resembling the one produced by a homogeneous sphere with infinite and zero impedance, respectively. A gradual rotation of the external electric field, or likewise a mechanical rotation of the nanoparticle, would surprisingly produce a gradual variation of its overall surface impedance, even if the materials that compose it are not varied. The extreme variation of its impedance is even more interesting considering that the materials composing the nanoparticle are not conductive. This behavior is clearly associated with the anomalous plasmonic resonance at the interface between the two plasmonic materials.

At the center of the resonant nanosphere, a singular concentration of the fields is observed, analogous to what we found in the 2-D case [6-7], associated to the plasmonic resonance at the interface of the hemispheres, and consistent with the subwavelength focusing properties of plasmonic materials. Panels $\mathrm{c}$ and $\mathrm{d}$ refer to the same configurations as in panel a and $b$, but now adding mismatch and realistic losses to the nanoparticle. In this case, the evaluation of the potential has been obtained numerically, since there is no closed-form solution to this problem in the general case. It can be seen how, despite the mismatch and absorption, the nanoswitching properties caused by rotation of the nanoparticle are still well preserved. 

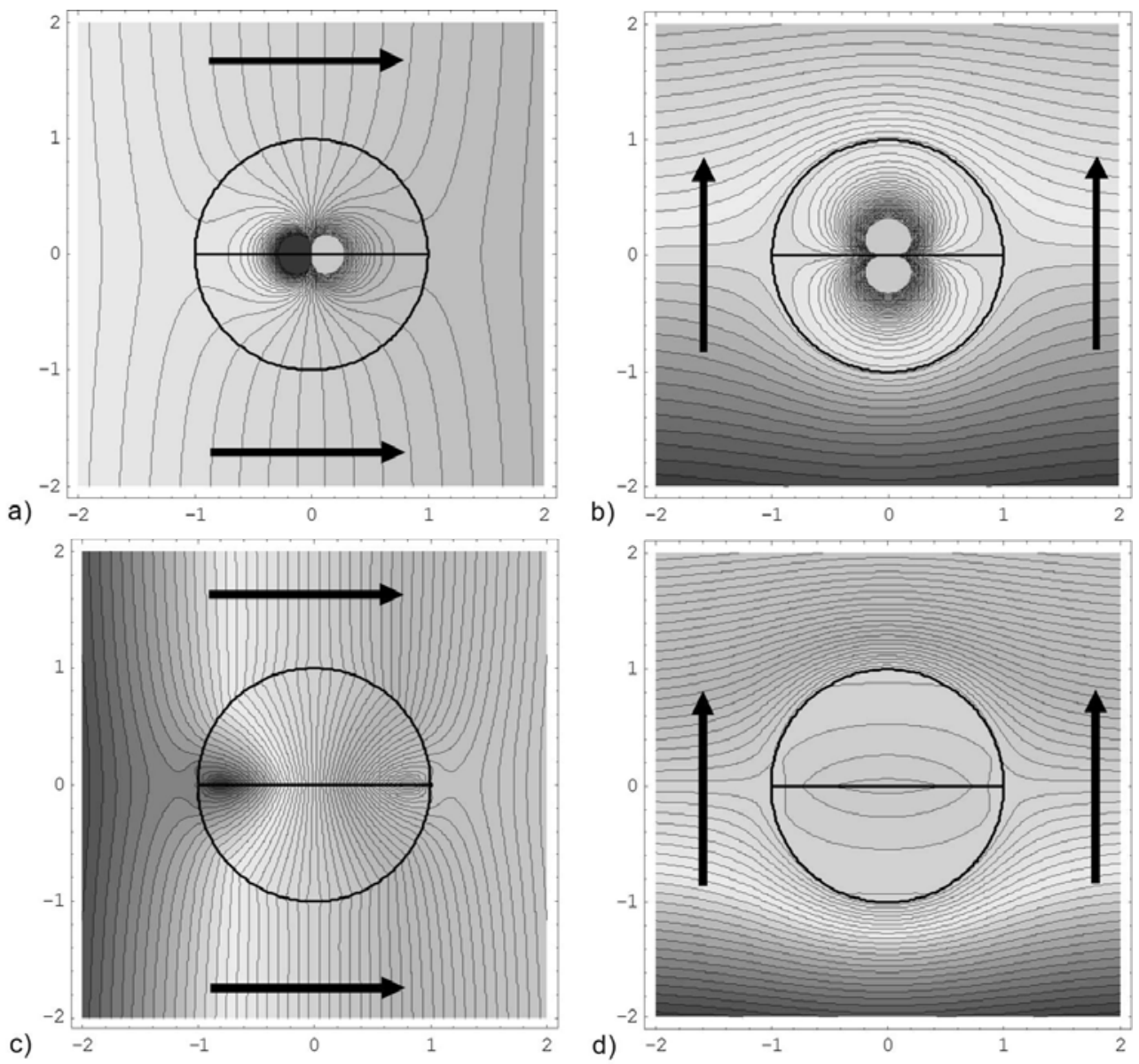

Figure 1 - Potential distribution around and inside the 3-D plasmonic spherical nanoswitch at resonance in the ideal case $\left(\varepsilon_{1}=-\varepsilon_{2}=16 \varepsilon_{0}\right)$ for parallel (panel a) and series configuration (panel $\mathrm{b}$ ); introducing realistic losses and permittivity mismatch $\varepsilon_{2}=(-16.1-j 0.2) \varepsilon_{0}$ in the spherical particles for parallel (panel c) and series (d) configurations. Darker regions correspond to larger values.

We foresee interesting potential applications of the anomalous optical response of this nanoparticle in nanocircuits and in the realization of novel optical metamaterials and metasurfaces.

\section{References:}

[1] N. Engheta, A. Salandrino, and A. Alù, "Circuit Elements at Optical Frequencies: Nano-Inductors, Nano-Capacitors and Nano-Resistors," Phys. Rev. Lett., Vol. 95, 095504, August 26, 2005.

[2] A. Alù, and N. Engheta, "Optical Nano-Transmission Lines: Synthesis of Planar Left-Handed Metamaterials in the Infrared and Visible Regimes," J. Opt. Soc. Am. B, Vol. 23, No. 3, pp. 571-583, March 2006. 
[3] A. Alù, and N. Engheta, "Theory of Linear Chains of Metamaterial/Plasmonic Particles as Sub-Diffraction Optical Nanotransmission Lines," Phys. Rev. B, Vol. 74, 205436, Nov. 29, 2006.

[4] A. Alù, and N. Engheta, "Three-Dimensional Nanotransmission Lines at Optical Frequencies: a Recipe for Broadband Negative-Refraction Optical Metamaterials," Phys. Rev. B, Vol. 75, 024304, Jan. 19, 2007.

[5] A. Alù, and N. Engheta, "Optical 'Shorting Wires'," Opt. Expr., Vol. 15, No. 21, pp. 13773-13782, Oct. 2007.

[6] A. Salandrino, A. Alù, and N. Engheta, "Parallel, Series, and Intermediate Interconnections of Optical Nanocircuit Elements - Part 1: Analytical Solution,” J. Opt. Soc. Am. B, Vol. 24, No. 12, pp. 3014-3022, Dec. 2007.

[7] A. Alù, A. Salandrino, and N. Engheta, "Parallel, Series, and Intermediate Interconnections of Optical Nanocircuit Elements - Part 2: Nanocircuit and Physical Interpretation,” J. Opt. Soc. Am. B, Vol. 24, No. 12, pp. 3007-3013, Dec. 2007.

[8] A. Alù, M. Young, and N. Engheta, "Nanofilters for Optical Nanocircuits," Phys. Rev. B, under review, online at: http://arxiv.org/abs/0710.0616.

[9] N. Engheta, "Circuits with light at nanoscales: optical nanocircuits inspired by metamaterials," Science, Vol. 317, 1698-1702, 2007.

[10] A. Alù, and N. Engheta, "Plasmonic Resonant Optical Nanoswitch," under review, online at: http://arxiv.org/abs/0710.4895. 